\title{
TIPOS DISCURSIVOS A LA BASE DE LA EDUCACIÓN FAMILIAR MAPUCHE $^{1}$
}

\author{
Discursive to base types of family education Mapuche \\ Daniel Quilaqueo* \\ César Aníbal Fernández ** \\ Segundo Quintriqueo ***
}

\section{RESUMEN}

El objetivo de este artículo es analizar cuatro tipos discursivos que se utilizan en la educación entregada por las familias en comunidades mapuches. Se realiza desde la perspectiva mapuche, respecto de categorías discursivas orales en la educación familiar, incorporando los antecedentes existentes en la investigación académica. El corpus sobre el cual se trabaja fue recopilado, en entrevistas realizadas a padres de familia y kimches (sabios). Los resultados muestran el análisis de los siguientes tipos de discurso orales: gübam, pentukün, ülkantun y piam.

Palabras clave: lengua oral, tipos discursivos, educación mapuche, memoria social.

\footnotetext{
1 Este artículo presenta resultados del proyecto Fondecyt Regular No 1140562, titulado: "Construcción social del conocimiento educativo mapuche: doble racionalidad y desafíos para una escolarización intercultural”.

* Centro de Investigación en Educación en Contexto Indígena e Intercultural, Facultad de Educación, Universidad Católica de Temuco. Temuco, Chile. Correo electrónico: dquilaq@uct.cl ** Facultad de Ciencias de la Educación, Universidad Nacional del Comahue. Neuquén, Argentina. Correo electrónico: cesaranibal@ciudad.com.ar.

*** Centro de Investigación en Educación en Contexto Indígena e Intercultural, Facultad de Educación, Universidad Católica de Temuco. Temuco, Chile. Correo electrónico: squintri@uct.cl
}

Artículo recibido el 26 de agosto de 2016. Aceptado el 11 de enero de 2017. 


\begin{abstract}
The aim of this article is to analyze some discourse types used in the education provided by the Mapuche families in the communities. This is done from a Mapuche perspective, regarding the oral discourse categories in Mapuche education, incorporating the existing backgrounds in academic research. The corpus used was collected via interviews to parents and kimches. The results show the analysis of these oral discourse types: gübam, pentukün, ülkantun and piam.
\end{abstract}

Keywords: Mapuche, ethnopolitics, municipality, fisheries legislation, ethnography.

\title{
INTRODUCCIÓN
}

El contexto cultural en el que se plantea la educación mapuche, en este artículo, es el de la oralidad en comunidades de la Araucanía en Chile. Su población presenta las características de que, por un lado, se encuentra escolarizada, accede a Internet y a los sistemas modernos de comunicación y, por otro lado, mantiene prácticas ancestrales específicas de su cultura. Aquí, se indaga la educación proporcionada por padres de familia, en relación con la oralidad, en forma separada de la escritura. Para ello, partimos de la hipótesis que la mayoría de los niños y jóvenes mapuches presentan dos lógicas de pensamiento en sus discursos, que como objeto de estudio denominamos doble racionalidad educativa que opera como soporte externo y concreto de la memoria social de cada familia y su comunidad; hoy día, necesaria para desarrollar la educación mapuche y escolar (Quilaqueo, et al., 2014-2017).

\section{ANTECEDENTES GENERALES}

Para este estudio se considera la tipificación de oralidad de Ong (1987) que establece dos categorías: una primaria y otra secundaria. La primera incluye a los pueblos sin escritura que recurren a la memoria y a la palabra para transmitir sus saberes. La segunda, en cambio, se apoya en la escritura que actúa como un recurso efectivo para recordar el conocimiento del pasado. Es decir, las sociedades alfabetizadas apelan a soportes tecnológicos para sostener el pasado. Las sociedades ágrafas, en cambio, recurren a la memoria social oral como soporte de sus conocimientos y como medio para transmitirlos y validarlos a través del tiempo (Montesperelli, 2004).

Actualmente aun hay pueblos que tienen -como apoyo de su educación, de sus creencias y de su pasado- a la memoria oral de la familia, es decir, la oralidad 
como soporte fundamental para el mantenimiento de su cultura. Para Vich y Zavala, "...la oralidad es una de las instancias mediante las cuales las sociedades construyen un archivo de conocimiento destinado a interpretar y negociar el pasado" (2004:18). El interés del valor de la oralidad, en este análisis, se apoya en la interpretación y negociación de los saberes y conocimientos propios, ya que históricamente, la primera función comunicativa del ser humano fue verbal.

La palabra oralidad es polisémica y su significado es motivo de controversias. El significado que se toma como referencia, para el estudio de la educación mapuche, es el de la lengua oral en el sentido social y cultural de los conocimientos que se transmiten por medio de relatos, cantos, discursos, consejos, cuentos, anécdotas y que son portadores de la tradición formativa de un pueblo (Montesperelli, 2004). De esta forma, se puede hacer referencia al hablante colectivo como voz de la comunidad que a través de la oralidad expresa la cultura de un pueblo (BlancheBenveniste, 1998).

Por otra parte, cabe agregar que la escritura históricamente ha estado vinculada con el poder. Ha sido empleada como símbolo y medio de dominación en la conquista de los pueblos nativos de América. Las rupturas entre la oralidad y la escritura se originan desde la llegada de los españoles; puesto que el uso del lenguaje ha sido "...también un medio de dominación y una fuerza social. Sirve para legitimar las relaciones de poder [donde] el lenguaje es también ideológico" (Wodak, 2003: 19). En consecuencia, el valor social de la oralidad ha estado dado por el hecho de que gran parte de las lenguas de los pueblos originarios americanos se han mantenido por el uso verbal.

Desde la literatura, Vargas Llosa sintetiza esta idea... "Porque hablar como habla un hablador es haber llegado a sentir y vivir lo más íntimo de esa cultura, haber calado en sus entresijos, llegado al tuétano de su historia y su mitología, somatizado sus tabúes, reflejos, apetitos y terrores ancestrales" (1987:188). Asimismo, Guevara (1908) empleaba el término 'hablador' para referirse a los relatores mapuches que utilizaban la palabra como reservorio verbal de la memoria colectiva de sus comunidades.

El pueblo mapuche reconoce personas que tienen la capacidad de recordar y transmitir los saberes de su pueblo. Tienen nombres específicos y se los llama kimche o sabio, machi o especialista del conocimiento medicinal, logko o jefe, weupife u orador; genpin hombre o mujer que en las rogativas 'lleva la palabra' haciendo oración por los demás y que dirige la acción tanto en el sacrificio de la colectividad como los particulares de cada familia. El werken es el mensajero o portavoz del logko y su comunidad de cuya memoria, antiguamente, dependía la fidelidad del mensaje. Las personas que desempeñan estos oficios son formados desde niño y la condición fundamental para ser elegido es tener buena memoria (Quilaqueo y Quintriqueo, 2010; Quilaqueo, 2013). 
La oratoria ha sido uno de los rasgos culturales mapuches más sobresaliente. Esta característica se ha mantenido en el tiempo y ha sido cultivada como un atributo propio y destacado. Lo seńala Cooper (1948) haciendo referencia a la prueba de oratoria que debían pasar los jóvenes. Lenz habla sobre dichas dotes retóricas en una cita que hace del Padre Rosales, quien en su Historia de Chile señala la preparación que se hacía en el campo de la oratoria de los nińos ejercitándolos en el uso de la palabra:

Dicen sus oraciones con tal rigor que parece que hablan con truenos y que sus oraciones son borrascas deshechas [...] es indecible cuán bien usan [...] de aquellas figuras de sentencias que encienden en los ánimos de los oyentes los afectos de la ira, indignación y furor que arden en el ánimo del orador, y a veces, los de lástima, compasión y misericordia, usando vivísimas prosopopeyas, hipótesis, reticencias irónicas, y de aquellas interrogaciones retóricas, no para preguntar, sino para reprender y argüir (Lenz, 1897: 6).

Por su parte, d'Orbigny resalta que para convertir al nińo en buen orador "la madre o hasta las ancianas lo acunan en sus primeros años contándole los altos hechos de sus antepasados muertos, haciendo el elogio de la elocuencia de que dieron muestra en las grandes ocasiones" (1945:842). Mansilla (1966), en su incursión a las comunidades mapuches en Argentina, trata el tema en varias partes de su obra. Hace referencia al 'lenguaraz o intérprete', a la relación entre lo ceremonial, lo lingüístico, lo proxémico y lo prosódico como parte destacable del contexto social mapuche. Analiza los distintos tipos de conversaciones: en familia y en parlamento. El entramado de la argumentación lo expresa marcando que pueden:

Convertir una razón en dos, en cuatro o más razones, quiere decir dar vuelta la frase por activa, y por pasiva, poner lo de atrás adelante, lo del medio al principio, o al fin; en dos palabras, dar vuelta a la frase de todos lados. El mérito del interlocutor en parlamento, su habilidad, su talento, consiste en el mayor número de veces que da vuelta a cada una de sus frases o razones... (Mansilla, 1966: 198).

Para el caso de Chile, Guevara señala que los weupife, eran personas formadas entre los mapuches que, además, poseían una memoria excepcional:

Tenían la profesión de recordar las genealogías de las familias en algunas reuniones, de pronunciar discursos, narrar episodios y transmitir mensajes de un grupo a otro. [...] Ejercían sus funciones de habladores sentados y a veces al compás de un tambor. Aunque era ocupación de los hombres, no estaban excluidas las mujeres. Gozaban todos marcadas consideraciones públicas (Guevara, 1908: 369-371). 
En resumen, la oralidad ha cumplido una variedad de roles que ha ido disminuyendo con el tiempo, pero que aún conserva funciones específicas en el desarrollo educativo y en la vida política y social de las comunidades. Debe seńalarse que al hacer referencia a la lengua hablada en la actualidad es necesario aclarar que se trata del empleo de dos lenguas: mapuche (mapunzugun) y castellano, con usos bilingües coordinados, subordinados o monolingües. Constituyen el principal pilar en el desarrollo del proceso educativo de los nińos, adolescentes y jóvenes mapuches (Quilaqueo et al., 2014).

\section{MÉTODO}

Este trabajo se enmarca en la perspectiva metodológica cualitativa y el método utilizado es el análisis crítico del discurso (Wodak, 2003). Históricamente, el uso del lenguaje, respecto del pueblo mapuche, ha sido un medio de dominación y una fuerza social que legitima las relaciones de poder mediante el discurso ideológico. El análisis crítico es una herramienta que permite visualizar la perspectiva de los padres de familia y kimches respecto de los conocimientos educativos desde su lógica cultural. Es decir, se interpreta el trasfondo ideológico presente en el lenguaje oral (Merino y Quilaqueo, 2003; Quilaqueo, 2006).

\section{Participantes}

El corpus de los discursos examina el relato de ocho padres de familia cuyas edades oscilaban entre los 50 y 80 años de edad, los participantes fueron considerados kimches por sus comunidades de las territorialidades Lafquenche (gente del borde costero del Océano Pacífico), Nagche (gente del valle) y Pehuenche (gente del sector cordillerano) pertenecientes a la región de la Araucanía en Chile. Todos los participantes accedieron a formar parte de la investigación de manera voluntaria.

\section{Instrumentos}

Los datos se obtuvieron a través de entrevistas semiestructuradas. Paralelamente se realizaron observaciones etnográficas que complementan el discurso del entrevistado. Para el análisis de los datos se consideró también la lógica del discurso en mapunzugun (Quilaqueo et al., 2012). El argumento metodológico de la entrevista semiestructurada se fundamenta en la saturación empírica de contenido (Pires, 1997). 


\section{RESULTADOS}

En los resultados se develan cuatro categorías de tipos discursivos mapuches: el gübam (consejo), pentukün (visita protocolar entre parientes), ülkantun (romanceadas) y piam (contar la memoria histórica). Estos tipos discursivos tienen por objeto describir e interpretar los modos de aprendizaje que ocurren en el medio familiar. Vale decir, las relaciones que se establecen entre los miembros de la comunidad, la dinámica grupal entre los niños, los mediadores que concurren en ese proceso, los reforzativos a que se recurre y la evaluación que van realizando de cada acción.

\section{Gübam}

En la educación mapuche se observa que el gübam tiene una finalidad didáctica, está dirigido a niños, adolescentes y jóvenes; cuyo ámbito es principalmente el hogar. La entrega del gübam se realiza normalmente entre padres e hijos, pero también puede serlo de abuelos a nietos. El rasgo fundamental es el consejo dado con afecto y autoridad. El vocablo es de antigua data, aparece registrado por Valdivia (1606:100) como "glamin" o "aconsejar" y en Augusta (1992: 67) gübam tiene igual significado. También, en estudios actuales, se usa el vocablo gübam como consejo dado a los niños (Quintriqueo, 2010). Es una estrategia a la que recurre la familia para enseñar tres tipos de valores: yamuwün que significa querer y valorar a las personas y a todos los seres; azmawün referido al respeto entre el medio natural y el hombre; y mañummawün o el agradecimiento hacia lo humano y no humano (Quilaqueo y Quintriqueo, 2010).

Para la enseńanza y aprendizaje hay varios tipos de gübam y están contextualizados de acuerdo con una edad determinada, básicamente en la infancia y adolescencia (Golluscio, 2006), en lugares cotidianos como el patio de la casa y en la hora posterior a las comidas. Esto ocurre también en sitios de celebración ritual y en ceremonias como el gijatun (rogativa) y we xipantu (año nuevo/nueva salida del sol), o en juegos como el palin (deporte similar al hockey). Un testimonio (Paine) indica que: "en las noches salen las mejores conversaciones; se les conversa a los niños, sentados junto al fogón, se recuerdan distintos temas o zugu; era lo que hacían los abuelos antiguamente". Esto significa que, en general, no hay un momento exclusivo y planificado, sino encuentros dados por las circunstancias y que varían según cada familia y lugar. Se trata de reuniones que ocurren en momentos de tranquilidad, donde se sientan y escuchan con atención.

Los responsables de hacer el gübam a los hijos son los padres y los abuelos. Se argumenta desde la vivencia de uno de nuestros entrevistados. 
Le decían: no deben ser personas malas ni ladronas ni apropiarse de lo ajeno; tienen que ser buenas personas; todo lo que aprendan les va servir a ustedes: es zapiluwün ["aprendizaje”]. Si estudias tienes que aprender a leer y escribir, de esa manera te van a respetar más los wigka (no mapuches); el que no aprende lo pasan a llevar y es engańado... Cuando el niño se distraía o se negaba a obedecer había un instrumento corrector: la varilla (Huerao Lepin).

Esta opinión muestra del choque cultural y la adopción de nuevas formas de educación. En este discurso subyace 'la dominación' cuando se señala “de esa manera te van a respetar más los wigka”. Además, refleja prácticas que pueden ser producto de la educación escolar colonial que se basó en el castigo para escolarizar a los mapuches, que se externaliza en la representación de los padres con respecto al acto de educar (Quintriqueo et al., 2014). En cambio el gübam es una herramienta metodológica empleada para la transmisión de los valores (Quilaqueo, 2006). Dentro del gübam se resalta la idea que engloba el concepto zapiluwün, definido como desarrollo de la capacidad de cultivarse y educarse que tienen las personas en base a la utilidad del consejo recibido (Quilaqueo y Quintriqueo, 2010). En otro testimonio, refiriéndose al tiempo pretérito, Paillán Huaiquil expresa que "en el lof mapu (comunidad) había un ñizol, una persona que era reconocida por todos como kimche; esa persona se caracterizaba por tener muy buena memoria y que sabía dar gübam; era muy respetado por toda la comunidad y siempre era tomada en cuenta su opinión”. Es decir, la memoria para recordar los contenidos formativos, de acuerdo a cada familia y comunidad, son aspectos fundamentales de quienes entregan el gübam.

Los niños no son los únicos destinatarios del gübam, los jóvenes también reciben recomendaciones, especialmente antes de contraer matrimonio. En general, los hijos e hijas aprenden observando y actuando junto a las personas mayores (padre, tíos, hermanos, abuelo) y de esa forma logran una identificación con su familia y comunidad (Quilaqueo y Quintriqueo, 2010). En síntesis, el gübam es un tipo de discurso oral, cuyo objetivo es formar a los niños, adolescentes y jóvenes en lengua mapuche -mapunzugun, en el ámbito hogareño, con respeto y solemnidad, pero también con afecto.

\section{Pentukün}

El pentukün es un tipo de discurso estructurado sobre la base de relaciones múltiples. Augusta (1991:37) registra un pentukün al que denomina "solemne salutación" que se produce entre una mujer que recibe a otra de una comunidad distinta de la propia. Catrileo (1995: 53) lo define como "visitar a alguien para felicitarlo, informarse sobre su salud o darle el pésame”. 
Por su parte, Malvestitti (2005: 29) indica que el pentukün "es un género específico, que a la vez constituye una secuencia introductoria en las conversaciones o parlamentos". En tanto, el testimonio de Millavil señala que "En el pentukün se conversa de cómo están las personas de un lof che en todos los ámbitos: salud, trabajo, enfermedad, muerte, sobre siembras y cosechas o el nacimiento de algún bebé. Pentukün es presentar saludos, felicitaciones, pésames; visitar a alguien por algún motivo especial".

La consultante Hueche Rañimán subraya que:

En la actualidad las personas solo se preguntan cómo está su salud y a eso le llaman pentukuvün. Antiguamente se visitaban para hacer pentukuvün y la persona que llegaba a una casa preguntaba ¿cómo has amanecido? y el dueño de casa respondía hemos amanecido bien, no hay problemas, nuestra familia está bien, mis hijos están bien. Y la persona que iba a visitar decía: yo he venido a saber cómo estabas, me ha enviado mi gran amigo (refiriéndose a un fücha che).

Esto muestra que en las conversaciones se hablaba sobre la familia, se aconseja a los adolescentes visitar sus familiares, para preguntar sobre la salud y eventuales necesidades, pesares y conflictos, como medio de formación de persona mapuche. Sin embargo, de acuerdo con lo develado en nuestros trabajos de campo, el pentukün también es un momento para compartir. Por ese motivo el contacto es prolongado y respetuoso. La noción de saludo aparece ligada con la de vínculo, ya que se saluda al amigo o al familiar y al que se aprecia. En resumen, puede decirse que se enmarca en la cortesía de tipo formal y solemne en la cual hay que presentar los saludos, felicitaciones o pésames, según la situación. Se trata de una relación social altamente estructurada.

\section{Ülkantun}

Los textos del ülkantun son expresados en lengua mapuche, en forma monódica y con intención poética por un hombre o una mujer en circunstancias sociales en las cuales suele primar la alegría; ya que el ülkantufe o cantor improvisa un texto o lo reproduce sobre la base de una transmisión comunitaria. El receptor suele ser del sexo opuesto que, en ocasiones, responde de inmediato creándose un contrapunto. A veces el mismo cantor entona la respuesta. Recibe distintos nombres, tales como ül, elegía, cantun. Según Augusta (1991:63) ülcantum significa "cantar algo". Se trata de una forma mapuche (Suárez, 1966) y no de un préstamo del español ni de una forma híbrida como suele sostenerse (Koessler, 1962). El ülkantufe es el cantor o intérprete.

Havelock (1996) dice que la poesía es producto de la oralidad y que para su mantenimiento y preservación aprovecha recursos auditivos o mnemónicos. 
Como ejemplo puede señalarse la preservación del ülkantun y del tayül que se han mantenido a través de los tiempos en la memoria de hablantes que no han accedido a la escritura (Fernández, 1995). El canto mapuche reconoce, básicamente, estas dos formas. Para nuestro trabajo nos ocuparemos únicamente del enunciado en primer término. El ülkantun puede tener una estructura fija o variable. Es fija cuando el texto se reitera sin cambios, mientras que se considera variable si cambia según la situación de improvisación. De esta forma se reconoce el ülkantun como un relato cantado sobre temáticas tales como dar la bienvenida a una persona que se considera importante, también una despedida o, en general, una visita y, en este sentido, puede ser parte del pentukün. También los son las declaraciones amorosas, elegías a la naturaleza, relatos sobre desgracias, canciones de machi para curar enfermedades, celebraciones deportivas y otras (Painequeo, 1991-1992). Salvo muy pocos textos, en general no hay referencias míticas o a hechos antiguos. El testimonio de Manquepi Cayul señala que "ülkantun es una canción que puede ser para recibir una visita, puede ser una canción después del juego del palín, puede ser un canto de victoria”.

Como texto poético en el ülkantun se recurre al empleo de tropos tales como metáforas, símiles, alegorías, metonimias e hipérboles. Painequeo (19911992) indica que hay ciertas partículas que se registran en numerosos textos y que adquieren el carácter de recurrentes, tales como ta, ga, anay, may, ti y que cumplen funciones eufónicas en el canto.

El ülkantun se lo conoce también con el nombre español de "romanceada". Ello se debe a la semejanza con la especie poética denominada romance que consistía en repetir los versos pares con la misma asonancia y los impares sin rima. Debe indicarse que la romanceada no tiene ningún tipos de rima y que tal recurso es desconocido en la cultura mapuche, salvo por el estribillo que suele usarse en algunos de esos textos. En el caso citado, se trata de una canción transcripta por Francisco Núñez de Pineda y Bascuñán, referida a hechos acontecidos en 1629 en Chile y que resulta ser la primera romanceada escrita en español. Dice:

La española y su hija me cogieron de las manos y llevaron en medio hasta el sitio adonde al son de sus alegres instrumentos bailaban y cantaban, y a su imitación, los que llegamos repetimos un romance que a mi despedida había compuesto (según supe) mi amigo y camarada Quilalebo, en nombre de su hija; que estando de la mano con ella, me dijo haber sido la compostura de la letra suya, porque mi ausencia le era de grande pesar y sentimiento (Francisco Núñez de Pineda y Bascuñán, 1863: 476).

El carácter bailado y al son de instrumentos se fue perdiendo y en la actualidad solo se entona a capella. En la Patagonia argentina se romancea, 
preferentemente, en la tarea-fiesta de la señalada o de la marcación de animales. En Chile, en cambio, los contextos son más amplios. Por último, debe señalarse que se trata, en efecto, de un tipo textual auténticamente mapuche que se ha mantenido por tradición oral y que solo recientemente se ha incorporado a la escritura en mapunzugun (Fernández, 1995).

\section{Piam}

Colicoy (2009), lo describe como un método que denomina piamtun, para hacer referencias a situaciones del pasado de la sociedad mapuche. Veamos un caso narrado por Marta Parra y citado por Colicoy (2009: 2).

La tierra era una gran casa de piedra. Allí vivía un matrimonio que tenía varios hijos: Küyen (luna), Antü (sol), Wünyelfe (lucero), Cherufe (ser no humano que no siempre es visible al hombre) y Che (gente, persona). Los padres salieron de su casa y los hermanos se pelearon; tan grande fue la pelea que no se fijaron que el fuego estaba encendido y que cada vez se hacía más grande, tanto que la casa explotó. Los hijos volaron por todas partes, unos hacia el espacio como Antü, Küyen y Wünyelfe; el Cherufe voló hacia unos montones de roca que ahora se le llama zegiñ (volcán); el Che era el menor voló junto a unos peñascos de piedra y ahí se quedó. Desde entonces dijeron: nunca más vamos a pelear, es mejor que trabajemos". Así Küyen trabaja regando, Antü hace fuego para temperar la casa, Cherufe vigila y limpia la casa todas las noches, recoge las cosas que ya no sirven. Él se alimenta sólo de carne humana, pero elimina a las personas que no se portan bien. Los seres humanos somos como los animales, algunos mansos, no son golosos, esos quedan para cría. Los mañosos y soberbios se venden o se matan para consumir.

El Cherufe elige Küyen, tuvo cuatro hijos: Tralkan (trueno), Lüfke (relámpago), Meulen (remolino) y Küef (viento). Cuando Küyen comenzó a regar, las piedras chicas y el Che se transformaron en hojas, las hojas en plantas y el Che comenzó a crecer igual que las plantas. Ahora que ustedes resucitaron, les dijo Küyen, ustedes no pueden vivir sin trabajar. Así que a Che lo nombraron como capitán y las plantas dejaron un camino por donde pasara el agua. Por eso somos hermanos, primos se puede decir. Cada uno de nosotros tiene su tierra, nadie lo puede molestar. Así también cada uno tiene su propio trabajo. Cuando no se respeta al otro surgen las discusiones, a pesar de que dijeron que no pelearían más, pero así es la vida. Por muy hermanos que seamos igual peleamos. Ellos son iguales, solo que Che tiene que calmar los ánimos. Por eso hay que hacer gijatun.

En este texto hay que diferenciar, por un lado, lo referido a un relato de creación que era desconocido por la literatura específica; en el mismo se cuenta el origen del universo como un Big Bang, donde el hombre es el ordenador del mundo terrestre y en el que se establece el vínculo del ser humano con el 
entorno. Por otro lado, se trata de un género discursivo, piam, que puede ser adoptado con fines didácticos en el ámbito escolar o doméstico para enseñar el valor la biodiversidad. En tanto, para Huenchucoy "el piam es un günen zugu (algo verdadero). Sin embargo, el mismo dice haber escuchado que también es un weza zungu (conocimiento maligno) decían personas de otros lugares... también decían que es un gübam malo, un gübam de "pillerías".

El concepto resulta confuso, ya que se lo asimila al epew, Colicoy (2009:3) utiliza con fines didácticos el término piamtun que “...presenta como un relato que no debe ser narrado en sí mismo, sino que tiene la intervención del narrador que levanta las enseńanzas del mundo físico, del mundo animal, del mundo vegetal y de las personas". Es decir, la acción de los personajes aparece como modelo para orientar a los niños, niñas, jóvenes y adultos mediante la analogía de personajes o entre animales como estrategia de enseñanza aprendizaje de los saberes mapuches.

\section{DISCUSIÓN Y CONCLUSIÓN}

En el estudio de la educación mapuche, este trabajo ha analizado la oralidad en forma separada de la escritura. En el estudio del discurso mapuche con sus reglas, sus tipologías y variabilidad de contenido muestra no solo una orientación hacia una oralidad afirmada a través de los tiempos, sino también su utilización precisa en la organización de la vida social y educativa (Quilaqueo y Quintriqueo, 2010). Esto incluye una tradición centenaria existente acerca del valor de la palabra mapuche, del mantenimiento de hábitos sociales que le han dado cohesión como pueblo (Quilaqueo, 2012). Son tipos de discursos a través de los cuales se mantuvo el conocimiento en la oralidad y a través de los que la educación ha permitido la formación de los niños y jóvenes. En esta categoría, en la sociedad mapuche contemporánea, están las personas reconocidas como sabias o kimches porque pueden acceder a la memoria social de la sociedad, en tanto 'hablante colectivo' y desde ese punto de vista se los puede incluir en la categoría de herederos de la oralidad primaria. En este sentido, la escritura no ha sido fundamental ni determinante para el desarrollo de la sociedad mapuche. Solo en la época contemporánea de los siglos XX y XXI ha adquirido una cierta relevancia.

En el desarrollo de la sociedad mapuche, si se analiza desde el punto de vista diacrónico, podrá decirse que existió una época de oralidad 'pura' que abarcó el tiempo en que solo había mapuches (Carrasco, 1990). En el contacto con los españoles primero y con chilenos y argentinos desde la independencia de ambos países hasta el presente, se vincularon con otra lengua y con la escritura de esa lengua, incluso con el latín empleado por los misioneros (de Valdivia, 1606). A partir de ese momento de relación y en el que los mapuches comenzaron a hablar 
castellano y a leer y escribir en ese idioma, cambiaron las formas comunicativas. La escritura fue adquiriendo cada vez más un rol específico y de una oralidad pura se fue pasando a la oralidad secundaria, primero a través de la escritura en castellano y actualmente, también con la escritura en mapunzugun. Consecuentemente, ya no puede hablarse de un sistema comunicacional que sea exclusivamente oral, sino que comparte algunos aspectos con la escritura; cada vez más y ahora también con los sistemas informáticos (Quilaqueo et al., 2014).

Podemos finalizar planteando que el desarrollo cultural de esta sociedad está actualmente encabalgada entre la oralidad y la escritura; lo que se explicaría desde la doble lógica educativa recibida: la educación entregada por la familia mapuche y por la escuela.

\section{REFERENCIAS}

Augusta, Félix de. Lecturas araucanas. Temuco: Editorial Kushe, 1991.

Augusta, Félix de. Diccionario mapuche-español y español mapuche (Tomos 1 y 2). Santiago, Ediciones Séneca, 1992.

Blanche-Benveniste, Claire. Estudios lingüisticos sobre la relación entre oralidad y escritura. Barcelona: Gedisa, 1998.

Carrasco, Iván. "Etnoliteratura mapuche y literatura chilena: relaciones", Actas de Lengua y Literatura Mapuche 4 (1990): 19-27.

Catrileo, María. Diccionario lingüistico-etnográfico de la lengua mapuche. MapudungunEspañol-Inglés. Santiago de Chile: Editorial Andrés Bello, 1995.

Colicoy, Domingo. Los vegetales en el mapuche kimvn. Disponible en: http://www. mapunet.org/documentos/mapuches/vegetales pdf, 2009. [Consultado el 7 de julio de 2014].

Cooper, John. "The Araucanians”. In Steward, J. (ed.). Handbook of South American Indians, T.II. Washington: Smithsonian Institution Bureau of American Ethnology, (1948): 687-760.

Fernández, César. Cuentan los mapuches. Antología. Buenos Aires: Ediciones Nuevo Siglo, 1995. 
Golluscio, Lucía. El pueblo mapuche: poéticas de pertenencia y devenir. Buenos Aires: Editorial Biblos, 2006.

Guevara, Tomás. Psicolojía del pueblo araucano. Santiago de Chile: Imprenta Cervantes, 1908.

Havelock, Eric. La musa aprende a escribir. Relaciones sobre oralidad y escritura desde la Antigüedad hasta el presente. Barcelona: Paidós, 1996.

Koessler-Ilg, Berta. Tradiciones araucanas. Tomo I. Universidad Nacional de La Plata: La Plata, 1962.

Lenz, Rodolfo. “De la literatura araucana”, Revista del Sur, año 1/7 (1897): 44.

Marisa (compiladora). Kiñe Rakizuam. Textos Mapuche de la Línea Sur. Universidad de Buenos Aires. Colección Nuestra América, 2005.

Mansilla, Lucio. Una excursión a los indios ranqueles. Buenos Aires: Editorial Kapelusz, 1966.

Merino, María y Quilaqueo, Daniel. "Ethnic prejudice against Mapuche in the discourse of members of Chilean society as a reflection of the racialist ideology of the Spanish conqueror", American Indian Cultural and Research Journal, 27/4, (2003): 15-24.

Montesperelli, Paolo. Sociología de la memoria. Buenos Aires: Ediciones Nueva Visión, 2004.

Núñez de Pineda y Bascunán, Francisco. (1863). Cautiverio feliz y razón de las guerras dilatadas de Chile. Santiago: Imprenta del Ferrocarril. Tomo III. Códice de 1673: 472-477.

Ong, Walther. Oralidad y escritura. Tecnologias de la palabra. México: Fondo de Cultura Económica, 1987.

Painequeo, Héctor. "Características del ül". Cuadernos de Lengua y Literatura, 3 y 4(1991-1992): 45-56.

Pires, Alvaro. "Échantillonnageet recherchequalitative: essaie théoriqueet métodologique". Poupart, Desloriers, Groulx, Lapièrre, Mayer, Pires (eds.). La recherche qualitative. Canada: Gaëtan Morin Editeur, (1997): 113-169. 
Quilaqueo, Daniel. "Les savoirs éducatifs mapuches. Des défis pour les enseignants” en Maheux et Gauthier, La formation des enseignants inuit et des premières nations. Problématiques et pistes d'action. Presses de l'Université du Québec, (2013): 107-124.

Quilaqueo, Daniel. "Saberes educativos mapuche: racionalidad apoyada en la memoria social de los kimches", Revista Atenea, 505(2012): 70-102.

Quilaqueo, Daniel. "Valores educativos mapuches para la formación de persona desde el discurso de kimches", Estudios Pedagógicos 32/2 (2006): 73-86.

Quilaqueo, Daniel; Quintriqueo, Segundo; Riquelme, Enrique; Loncón, Elisa y Le Bonniec, Fabien. "Construcción social del conocimiento educativo mapuche: doble racionalidad y desafíos para una escolarización intercultural”, Proyecto Fondecyt Regular N 1140562, 2014-2017.

Quilaqueo, Daniel; Quintriqueo, Segundo; Torres, Héctor y Muñoz, Gerardo. "Saberes educativos mapuches: aportes epistémicos para un enfoque de educación intercultural", Chungara Revista de Antropología Chilena 46/2 (2014): 271-283.

Quilaqueo, Daniel y Quintriqueo, Segundo. "Saberes educativos mapuches: un análisis desde la perspectiva de los kimches”, Revista Polis, 26 (2010): 337360.

Quintriqueo, Segundo. Implicancias de un modelo curricular monocultural en contexto mapuche. Temuco: Universidad Católica de Temuco, 2010.

Suárez, Jorge. "Indigenismos e hispanismos vistos desde la Argentina”, Romance Philology, 20/1(1966): 68:90.

Vargas Llosa, Mario. El hablador. Barcelona: Seix Barral, 1987.

Valdivia, Luis de. (1606). Arte y Gramática de la lengua que corre por todo el Reyno de Chile, con su Vocabulario, y Confesionario. Lima.

Vich, Víctor y Virginia Zavala. Oralidad y poder. Herramientas metodológicas, Bogotá: Norma, 2004. 
Wodak, Ruth. "De qué trata el análisis crítico del discurso (ACD). Resumen de su historia, sus conceptos fundamentales y sus desarrollos". En Wodak, R. y Meyer, M. (comps.). Métodos de análisis crítico del discurso. Barcelona, España: Editorial Gedisa, 2003. 